Publ. RIMS, Kyoto Univ.

41 (2005), 971-977

\title{
A Double Exponential Formula for the Fourier Transforms
}

By

Takuya OourA*

\section{$\S 1 . \quad$ Introduction}

In this paper, we propose a new and efficient method that is applicable for the computation of the Fourier transform of a function which may possess a singular point or slowly converge at infinity. The proposed method is based on a generalization of the method of the double exponential (DE) formula; the DE formula is a powerful numerical quadrature proposed by H. Takahasi and M. Mori in 1974 [1]. Although it is a widely applicable formula, it is not effective in computing the Fourier transform of a slowly decreasing function. Actually it is not very efficient even if one wants to compute the value of a Fourier transform at a particular point, i.e., a Fourier-type integral. To conquer this weakness at least for a Fourier-type integral, M. Mori and the author proposed a new DE formula in 1991 [2]. See also [3] for a further improvement. The method proposed there is effective for Fourier-type integrals, but it is still weak in the computation of the Fourier transform. Here we propose another DE formula which is applicable to the computation of the Fourier transform. One point in the new method proposed here is that it makes use of fixed sampling points even if we change the point where the Fourier integral is evaluated.

In this paper we propose the new method and illustrate the efficiency of the new method in several concrete examples through the comparison with the older methods.

Communicated by H. Okamoto. Received August 5, 2005.

2000 Mathematics Subject Classification(s): 65D32, 65T50, 65Y20.

Partially Supported by Grant-in-Aid of JSPS, No. 16760054.

*RIMS, Kyoto University, Kyoto 606-8502, Japan. 


\section{$\S 2 . \quad$ DE Formula for Integrals}

We first review the classical DE formula for integrals. For the sake of simplicity, we restrict ourselves to the following integral including a sine function and a parameter $\omega>0$

$$
I=\int_{0}^{\infty} f(x) \sin \omega x \mathrm{~d} x
$$

assuming that $f(x)$ converges very slowly as $x \rightarrow \infty$. We apply to (2.1) a variable transformation

$$
x=M \varphi(t), \quad \varphi(t)=\frac{t}{1-\exp \left(-2 t-\alpha\left(1-\mathrm{e}^{-t}\right)-\beta\left(\mathrm{e}^{t}-1\right)\right)},
$$

where $M, \alpha$, and $\beta$ are positive constants, and obtain

$$
I=\int_{-\infty}^{\infty} f(M \varphi(t)) \sin (\omega M \varphi(t)) M \varphi^{\prime}(t) \mathrm{d} t .
$$

Applying the trapezoidal rule with mesh size $h$, we have

$$
I_{h}=M h \sum_{n=-\infty}^{\infty} f(M \varphi(n h)) \sin (\omega M \varphi(n h)) \varphi^{\prime}(n h) .
$$

We assume that $M$ satisfies $\omega M h=\pi$. Then, $\sin (\omega M \varphi(n h)) \rightarrow \sin n \pi=0$ rapidly as $n \rightarrow \infty$ since $\varphi(t) / t \rightarrow 1$ as $t \rightarrow \infty$, and (2.4) converges to $I$ in (2.3) very quickly. Thus, the following formula is obtained [3].

Approximation Formula 1. Choose $h>0$ and two integers $N_{+}$and $N_{-}$. Compute

$$
I_{h}^{(N)}=\frac{\pi}{\omega} \sum_{n=-N_{-}}^{N_{+}} f\left(\frac{\pi}{\omega h} \varphi(n h)\right) \sin \left(\frac{\pi}{h} \varphi(n h)\right) \varphi^{\prime}(n h),
$$

where the parameters of $\varphi(t)$ are chosen as $\alpha=\beta / \sqrt{1+\log (1+\pi /(\omega h)) /(4 \omega h)}$, $\beta=0.25$. If $N=N_{-}+N_{+}+1$ is appropriately chosen, the error is bounded as $\left|I-I_{h}^{(N)}\right|<c^{\prime} \exp (-c / h)<C^{\prime} \exp (-C N / \log N)$ for typical $f$ 's, where $c, c^{\prime}, C, C^{\prime}$ are positive constants depending only on $f(x)$ and $\omega$.

The integrand and the sampling points in the case of $f(x)=x^{-1 / 2}$ and $\omega=$ 1 are shown in Figure 1. We can see that the sampling points are dependent on the period $\pi / \omega$. We note that the change of $\omega$ forces the change of the sampling points. Hence, the computation on varying $\omega$ 's of the Fourier transform using Approximation Formula 1 requires many sampling points. 


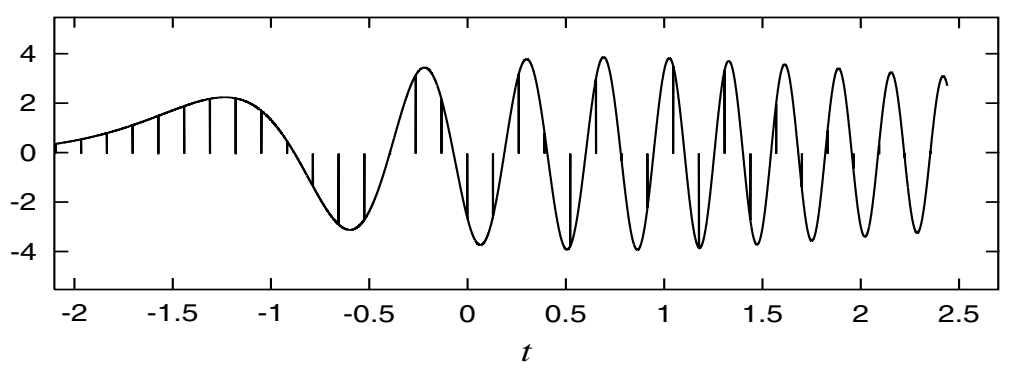

Figure 1. Integrand of (2.3) and sampling points of (2.4).

\section{§3. DE Formula for Transforms}

We now propose a new method. We will derive an approximation formula for the Fourier transform

$$
F(\omega)=\int_{0}^{\infty} f(x) \exp (\mathrm{i} \omega x) \mathrm{d} x
$$

By applying the variable transformation (2.2), we obtain

$$
F(\omega)=\int_{-\infty}^{\infty} f(M \varphi(t)) \exp (\mathrm{i} \omega M \varphi(t)) M \varphi^{\prime}(t) \mathrm{d} t .
$$

Next we define

$$
E(\omega)=\int_{-\infty}^{\infty} f(M \varphi(t)) \exp \left(\mathrm{i} \omega M \varphi(t)-\mathrm{i} \omega_{0} M \hat{\varphi}(t)\right) M \varphi^{\prime}(t) \mathrm{d} t
$$

where $\hat{\varphi}(t)=\varphi(t)-t$ and $\omega_{0}$ is a positive constant to be fixed later. Then, $|E(\omega)|$ is very small for large $M$, and the order is

$$
|E(\omega)|=O\left(\exp \left(-d^{\prime} M \min \left(\omega, \omega_{0}\right)\right)\right)
$$

for typical f's, where $d^{\prime}$ is a positive constant depending on $f(x)$. As the proof of this fact is rather complicated, it will be given elsewhere. We calculate $\tilde{F}(\omega)=F(\omega)-E(\omega)$ instead of $F(\omega)$. Then,

$$
\begin{array}{r}
\tilde{F}(\omega)=\int_{-\infty}^{\infty} f(M \varphi(t)) \exp \left(\mathrm{i} \omega M \varphi(t)-\frac{\mathrm{i}}{2} \omega_{0} M \hat{\varphi}(t)\right) \\
\cdot 2 \mathrm{i} M \sin \left(\frac{1}{2} \omega_{0} M \hat{\varphi}(t)\right) \varphi^{\prime}(t) \mathrm{d} t
\end{array}
$$

Since $\hat{\varphi}(t) \rightarrow 0$ as $t \rightarrow+\infty$ and $\varphi^{\prime}(t) \rightarrow 0$ as $t \rightarrow-\infty,\left|\sin \left(\frac{1}{2} \omega_{0} M \hat{\varphi}(t)\right) \varphi^{\prime}(t)\right|$ converges to zero rapidly as $t \rightarrow \pm \infty$. Therefore the integrand of $\tilde{F}(\omega)$ is a 
function to which we can readily apply the ordinary DE method. This means that we can choose the mesh size $h$ independently of $\omega$. As an illustration, the imaginary parts of the integrands of $F(\omega), E(\omega)$ and $F(\omega)-E(\omega)$ in the case of $f(x)=x^{-1 / 2}$ are shown in Figure 2. To compute integral for $\omega \in\left(0,2 \omega_{0}\right)$, it suffices to choose $M=\pi /\left(\omega_{0} h\right)$. Then, the following formulas are obtained.

Approximation Formula 2. Let us compute the integral (3.1) for $\omega$ in the interval $\left(0,2 \omega_{0}\right)$. Then, the approximation formula we propose is as follows:

$$
\begin{gathered}
\tilde{F}_{h}^{(N)}(\omega)=\frac{2 \pi \mathrm{i}}{\omega_{0}} \sum_{n=-N_{-}}^{N_{+}} f\left(\frac{\pi}{\omega_{0} h} \varphi(n h)\right) \sin \left(\frac{\pi}{2 h} \hat{\varphi}(n h)\right) \varphi^{\prime}(n h) \\
\cdot \exp \left(\frac{\pi \mathrm{i} \omega}{\omega_{0} h} \varphi(n h)-\frac{\pi \mathrm{i}}{2 h} \hat{\varphi}(n h)\right),
\end{gathered}
$$

where the parameters of $\varphi(t)$ is chosen as $\alpha=\beta / \sqrt{1+\log \left(1+\pi /\left(\omega_{0} h\right)\right) /\left(4 \omega_{0} h\right)}$, $\beta=0.25$. This formula is approximated in the interval $0<\omega<2 \omega_{0}$, and the error is bounded by $\left|F(\omega)-\tilde{F}_{h}^{(N)}(\omega)\right|<c_{0}^{\prime} \mathrm{e}^{-c_{0} / h}+c_{1}^{\prime} \mathrm{e}^{-c_{1} \omega / h}+c_{2}^{\prime} \mathrm{e}^{-c_{2}\left(2 \omega_{0}-\omega\right) / h}$, where $c_{i}, c_{i}^{\prime}$ are positive constants depending on $f(x)$ through $d^{\prime}$.

Once $\omega_{0}$ is fixed, the sampling points of Approximation Formula 2 need not be changed. Moreover, the imaginary part of Approximation Formula 2 in the case of $\omega=\omega_{0}$ is the same as Approximation Formula 1. Therefore, Approximation Formula 2 is a generalization of Approximation Formula 1.
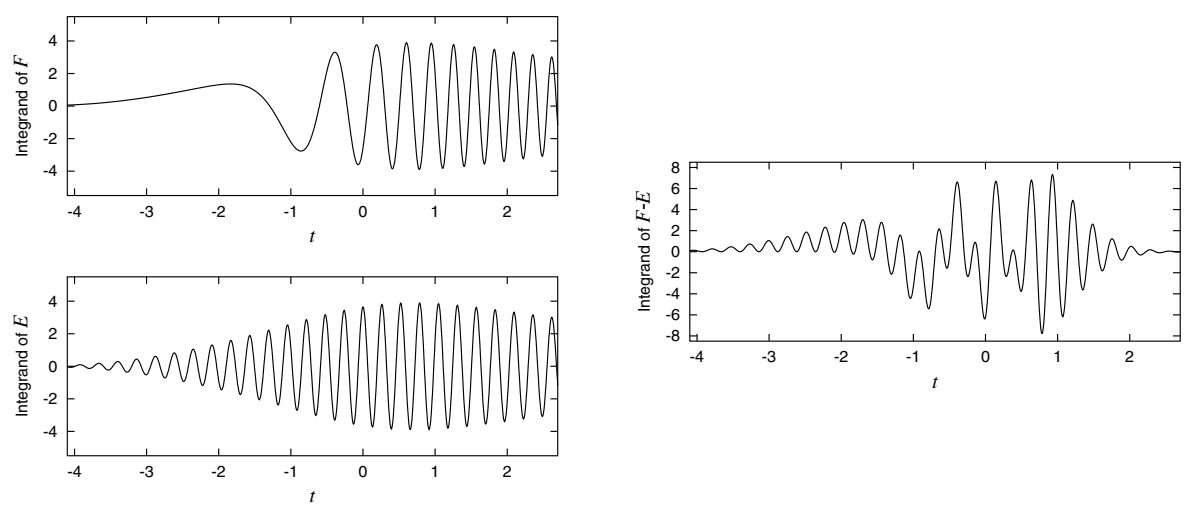

Figure 2. Integrands of (3.2), (3.3), (3.4). 


\section{$\S 4$. A Numerical Example}

To illustrate the effectiveness of the proposed method, we consider the following integral:

$$
F(\omega)=\int_{0}^{\infty} \frac{\log x}{\sqrt{x}} \exp (\mathrm{i} \omega x) \mathrm{d} x,
$$

which cannot be computed efficiently by the FFT-based method since the integrand has a singularity at $x=0$ and decays very slowly as $x \rightarrow \infty$. Figure 3 shows the absolute error of the computation by using Approximation Formula 2 with parameters $\omega_{0}=1, h=0.075, N_{-}=94, N_{+}=69$. In the parameters, the interval of effective accuracy is $0<\omega<2 \omega_{0}=2$. The computation was done in double precision (53-bit precision) so the round-off error is about $10^{-15}$.

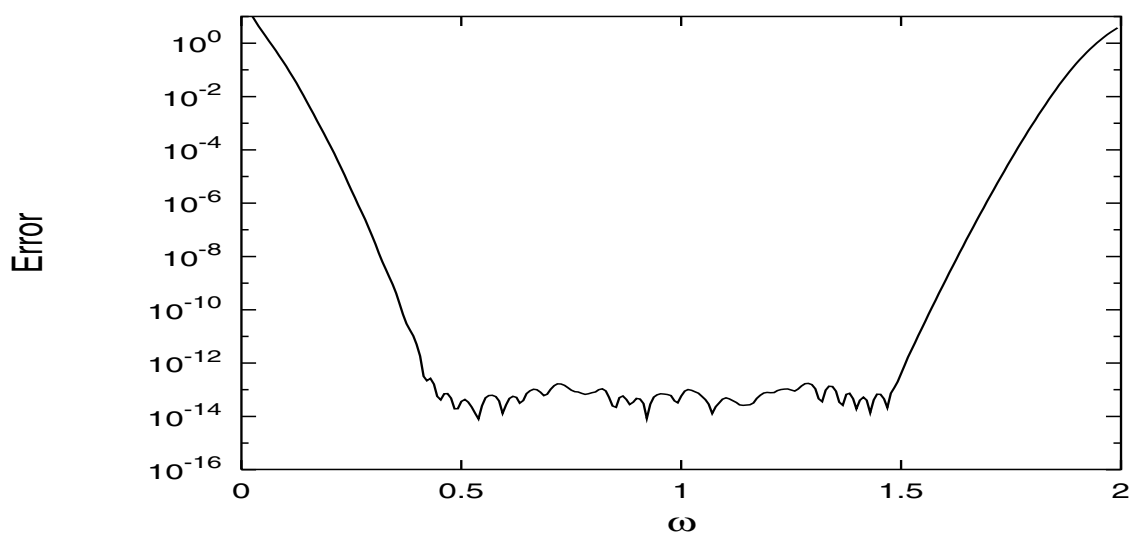

Figure 3. $\left|F(\omega)-\tilde{F}_{h}^{(N)}(\omega)\right|,\left(h=0.075, N_{-}=94, N_{+}=69\right)$.

Figure 4 and Figure 5 show the absolute error when the mesh size $h$ is changed on the same computation environment. We observe that the interval of effective accuracy becomes larger if the mesh size $h$ is reduced. Here we note the following: when the mesh size $h$ is reduced the accuracy improves but bigger $N_{+}$and $N_{-}$are needed. To compute for a wider range of $\omega$, the method of changing $\omega_{0}$ and the re-computation is recommended.

\section{$\S 4.1$. Comparison with a conventional method}

Let us compare the labor we need to compute (4.1) approximately by our new method and that by the conventional DE method: we want to compute 


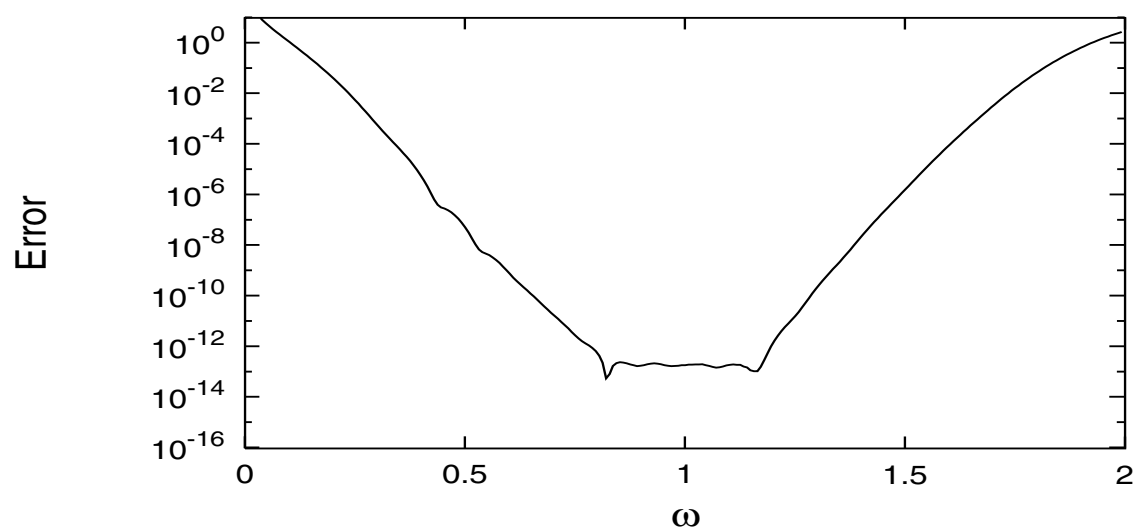

Figure 4. $\left|F(\omega)-\tilde{F}_{h}^{(N)}(\omega)\right|,\left(h=0.15, N_{-}=44, N_{+}=35\right)$.

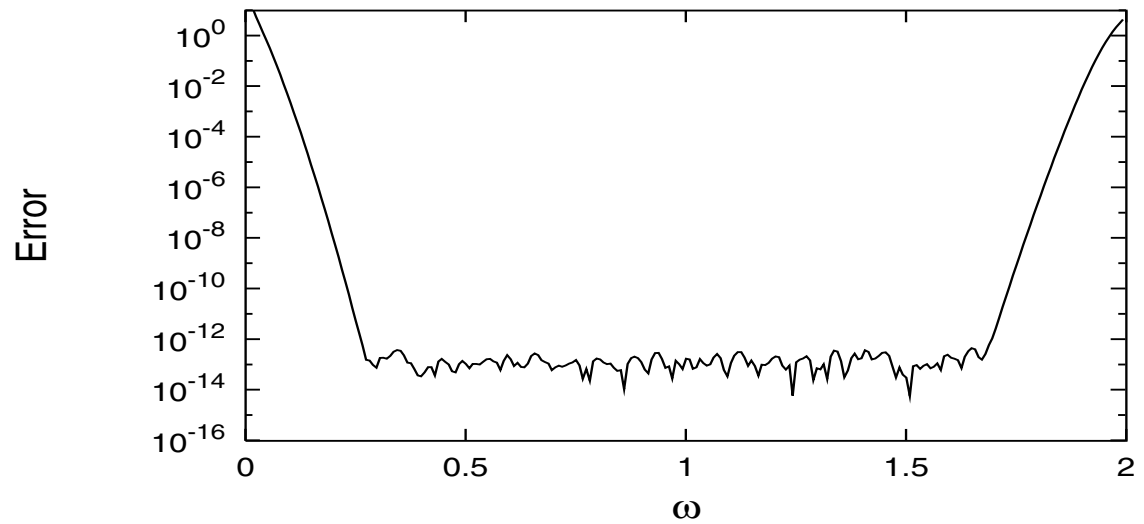

Figure 5. $\left|F(\omega)-\tilde{F}_{h}^{(N)}(\omega)\right|,\left(h=0.03, N_{-}=253, N_{+}=173\right)$. 
(4.1) with the absolute error tolerance $10^{-12}$ and the interval of $\omega$ is set to $0.5 \leq$ $\omega<1.5$. We computed 128-point transform $F(0.5+k / 128), k=0,1,2, \cdots, 127$. Table 1 shows the total number $N$ of the function evaluations and the execution time.

Table 1. Comparison of quadrature methods.

\begin{tabular}{|c|l|c|c|}
\hline & $h$ & $N$ & $\begin{array}{c}\text { CPU time } \\
\text { (Xeon 3.06GHz }+ \text { gcc3.2) }\end{array}$ \\
\hline Proposed DE (Formula 2) & 0.075 & 164 & $0.07 \mathrm{~m} \mathrm{sec}$ \\
DE Formula [3] (Formula 1) & 0.15 & 20096 & $2.42 \mathrm{~m} \mathrm{sec}$ \\
\hline
\end{tabular}

As another example, let us consider

$$
F(\omega)=\int_{0}^{\infty} \frac{1}{\sqrt{1+x^{2}}} \cos (\omega x) \mathrm{d} x .
$$

This time we also try another powerful method: the continuous Euler transformation [4] with FFT, which is applicable to (4.2). The absolute error tolerance is set at $10^{-12}$ and the interval of $\omega$ is set to $0.5 \leq \omega<1.5$. We computed 128 -point transform. Table 2 shows the result of the computation.

Table 2. Comparison of quadrature methods.

\begin{tabular}{|c|l|c|c|}
\hline & \multicolumn{1}{|c|}{$h$} & $N$ & $\begin{array}{c}\text { CPU time } \\
\text { (Xeon 3.06GHz }+ \text { gcc3.2) }\end{array}$ \\
\hline Proposed DE (Formula 2) & 0.075 & 157 & $0.04 \mathrm{~m} \mathrm{sec}$ \\
DE Formula [3] (Formula 1) & 0.15 & 9856 & $0.89 \mathrm{~m} \mathrm{sec}$ \\
\hline Continuous Euler Transf.+FFT & $\pi / 16$ & 2049 & $0.12 \mathrm{~m} \mathrm{sec}$ \\
\hline
\end{tabular}

\section{References}

[1] Takahasi, H. and Mori, M., Double exponential formulas for numerical integration, Publ. RIMS, Kyoto Univ., 9 (1974), 721-741.

[2] Ooura, T. and Mori, M., The double exponential formula for oscillatory functions over the half infinite interval, J. Comput. Appl. Math., 38 (1991), 353-360.

[3] - A robust double exponential formula for Fourier type integrals, J. Comput. Appl. Math., 112 (1999), 229-241.

[4] Ooura, T., A continuous Euler transformation and its application to the Fourier transform of a slowly decaying function, J. Comput. Appl. Math., 130 (2001), 259-270. 